\title{
From presentation to follow-up: diagnosis and treatment of cerebral venous thrombosis
}

\author{
J. Nicole Bentley, B.S., ${ }^{1}$ Ramón E. Figueroa, M.D., ${ }^{2}$ And John R. Vender, M.D. ${ }^{1}$ \\ Departments of ${ }^{1}$ Neurosurgery and ${ }^{2}$ Neuroradiology, Medical College of Georgia, Augusta, Georgia
}

\begin{abstract}
Cerebral venous thrombosis is an uncommon cause of stroke but remains a challenge for physicians faced with this diagnosis largely due to the variability in presentation. Anticoagulation, typically with intravenous heparin, remains the mainstay of treatment for stable patients and is sufficient in the majority of cases. However, a significant mortality rate exists for cerebral venous thrombosis due to patients who deteriorate or do not adequately respond to initial treatments. It is in these patients that more aggressive interventions must be undertaken. The neurosurgeon is often called on, either acutely for initial evaluation of the stroke or venous hemorrhage or after the failure of initial therapy for clot evacuation, hemicraniectomy, or thrombectomy. A proper workup must include a search for an underlying, correctable cause as well as thorough follow-up with correction of identified risk factors to decrease the risk of recurrent disease. (DOI: 10.3171/2009.8.FOCUS09166)
\end{abstract}

\section{KEY WoRds - cerebral venous thrombosis • anticoagulation • rheolysis - thrombectomy}

$\mathrm{C}$ EREBRAL venous thrombosis is rare, accounting for just $0.5 \%$ of all strokes. ${ }^{7}$ However, rapid and accurate diagnosis is paramount to patient safety and recovery. Overall mortality rates range from 5 to $30 \% .^{10}$ This can approach $50 \%$ in cases of treatment failure or when patients have evidence of clinical deterioration. ${ }^{21,40,49}$ In these more complicated situations, aggressive techniques to alleviate clot burden and subsequent intracranial mass effect are required..$^{7,10}$ A tertiary care center may see $\sim 5$ cases per year, and therefore, care providers must be knowledgeable about the current methods of diagnosis and treatment of CVT. ${ }^{6}$ Patients will often present initially to a stroke service, but neurosurgeons are often consulted for increasing ICP or hemorrhagic infarcts. Additionally, patients in whom conservative treatment with anticoagulation fails may need surgical intervention. ${ }^{49}$

Cerebral venous thrombosis predominantly affects young people who are otherwise healthy, and women are affected more often than men. ${ }^{10}$ Risk factors are varied, but virtually any medical condition that causes hypercoagulability and susceptibility to blood clotting should be considered. ${ }^{7,15,49}$ In a study of 31 patients, risk factors were identified in $94 \%$, and $65 \%$ had more than one risk factor. Women most commonly had associated oral contraceptive or estrogen replacement use, pregnancy, or puerpe-

\footnotetext{
Abbreviations used in this paper: $\mathrm{CVT}=$ cerebral venous thrombosis; $\mathrm{ICP}=$ intracranial pressure; $\mathrm{IIH}=$ idiopathic intracranial hypertension; rtPA = recombinant tissue plasminogen activator.
}

rium. The distribution among risk factors was equal in men, comprising germ cell malignancies, dehydration, and hematological disorders. Men were more likely to have 1 risk factor. ${ }^{49}$ Other frequently seen causes include Factor V Leiden, ${ }^{1}$ protein $\mathrm{C},{ }^{28}$ or protein $\mathrm{S}^{39}$ deficiencies; antiphospholipid syndrome; ${ }^{45}$ vasculitis; ${ }^{26}$ or infections. ${ }^{38}$ It has been suggested that pseudotumor cerebri is due to undiagnosed CVT, and one study has suggested that MR venography should be done unless MR imaging is diagnostic for CVT in any patient with presumed IIH, to rule out this condition. Lin et al. ${ }^{34}$ found that $9.4 \%$ of patients (10 of 106) meeting criteria for IIH had thrombosis evident on MR venography. However, the classic demographic for IIH (a young, obese female) was not sensitive enough to allow for MR venography in just this subset, as the diagnosis in 4 patients would have been missed. ${ }^{3}$ Considering that underlying malignancies causing a hypercoagulable state may be diagnosed once CVT is confirmed, the risk of not performing a thorough workup for IIH can be of significant detriment to the patient. ${ }^{6,34}$

\section{Diagnosis}

The diagnosis of CVT can present a substantial challenge. Misdiagnosis and delay in diagnosis are common problems, particularly in patients presenting to facilities without established stroke, neurological, or neurosurgical support. ${ }^{22}$ Initial misdiagnosis occurs in up to $73 \%,{ }^{49}$ and delay has been reported at longer than 10 days in $40 \%$ of patients. ${ }^{16}$ Signs and symptoms are extremely varied, and 


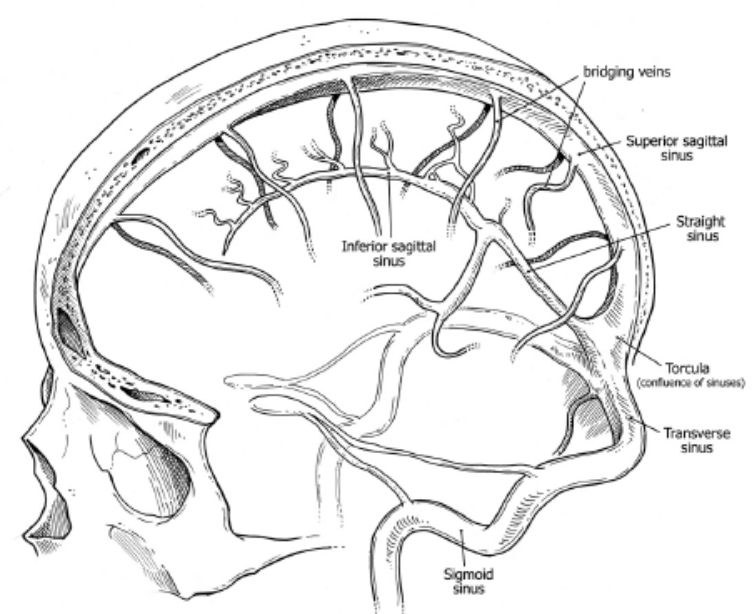

FIG. 1. Normal venous sinus anatomy in the cerebral hemispheres. (C)2009 Medical College of Georgia

none are pathognomonic. Headache is the most common symptom, occurring in up to $90 \%,{ }^{21}$ and can be identical to the "worst headache of my life," as described in subarachnoid hemorrhage. ${ }^{13}$ The presence of headache as a symptom is not insignificant, since this complaint alone is responsible for up to $16 \%$ of all emergency room visits, and is therefore among the most common reasons for a patient to present there. ${ }^{37}$ Physicians must be able to evaluate this symptom thoroughly and discern who requires further workup.

Although not specific for CVT, different sources report the incidence of some signs and symptoms that can be helpful in localization. Visual impairment occurs in $10-13 \%$ of patients and papilledema in $27-80 \%$ and can point to thrombus in the cavernous sinus; focal deficits have been reported in $27-79 \%$ and are suggestive of isolated cortical vein thrombosis. Superior sagittal sinus thrombosis can cause seizures, which has been observed in 10-63\% of patients, and coma was the presenting symptom in $15 \%$, pointing to involvement of the deep veins. Aphasia, suggestive of transverse sinus occlusion, occurs in $\sim 13 \%$ of patients. ${ }^{5,49}$ Figure 1 shows normal sinus anatomy and likely places for thrombosis to occur. Figure 2 shows an in vivo image of the late venous phase on angiography, with an area of flow deficit in the left transverse sinus.

There are other less common symptoms, such as nausea and vomiting, migraine, tinnitus, cranial nerve palsy, and psychiatric symptoms..$^{5-8,10,13,16,20,21,36}$ A syndrome of intracranial hypertension with headache occurs most frequently, and most people present with more than one symptom. The condition can mimic a variety of intracranial processes, including tumor, abscess, encephalitis, arterial stroke, and benign intracranial hypertension. ${ }^{6} \mathrm{~A}$ low threshold for workup should be maintained, and CVT should always be included in the differential diagnosis in the presence of vague neurological findings.

\section{Clinical Course and Prognosis}

Also confounding the diagnosis is the unpredictable

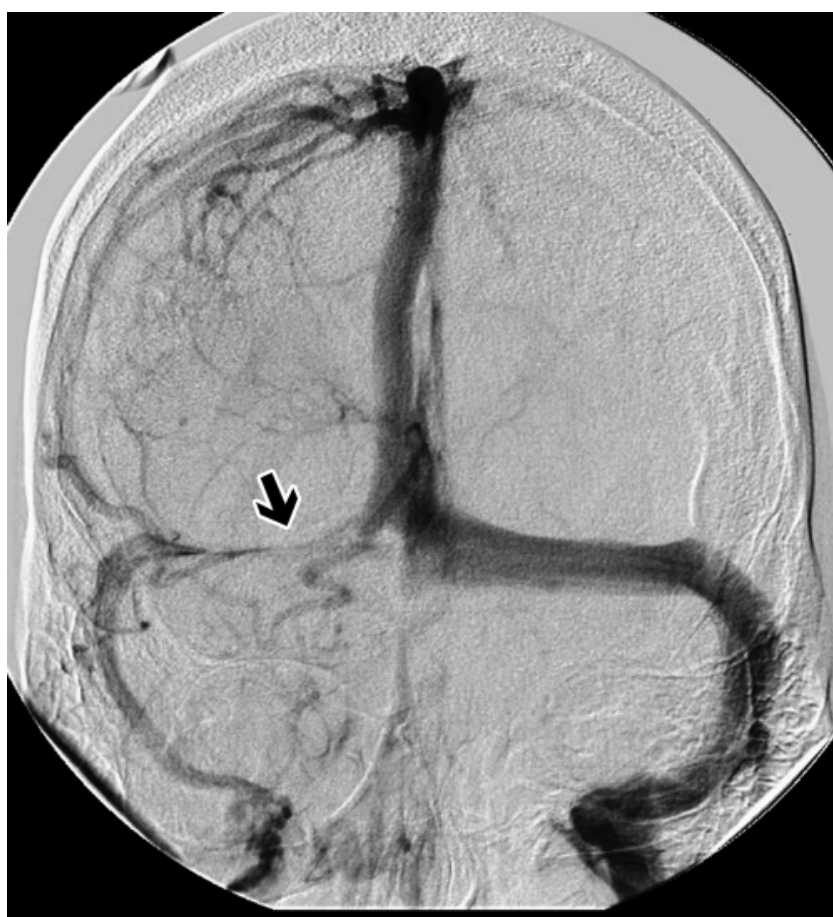

FIG. 2. Angiogram showing contrast in the late venous phase, with an area of flow deficit in the left transverse sinus (arrow) consistent with thrombosis. (02009 Medical College of Georgia

progression of symptoms, which, unlike arterial stroke, can range from a few hours to several months. Neither the rapidity of evolution nor the severity at presentation is predictive of ultimate outcome. Predicting who will fare worst is extremely difficult. Isolated headache can quickly deteriorate into coma or death, while a patient with multiple focal neurological deficits may recover completely. ${ }^{33}$ However, there is some guidance on poor prognostic characteristics. Investigators of the International Study of Cerebral Venous Thrombosis (ISCVT) determined the following 8 variables at presentation that correlate well with prognosis: age $>37$ years, male sex, altered mental status, hemorrhage on admission CT scan, coma, deep cerebral vein involvement, CNS infection, and malignancy. Coma at presentation remains the worst prognostic indicator overall. ${ }^{21}$ A viable collateral system of cortical and bridging veins is a key factor in the clinical presentation and mortality rate. An intact collateral system can significantly attenuate increases in ICP. However, if the thrombus extends to involve collateral circulation, local hypoperfusion with infarction and destruction of the blood-brain barrier and vasogenic edema can occur. ${ }^{4,40}$ Infarctions occur in $\sim 50 \%$ of cases. ${ }^{24}$ It is in these patients in whom more immediate and aggressive treatment is indicated.

The D-dimer assay, a familiar serum test useful in deep vein thrombosis, pulmonary emboli, and coronary artery thrombosis, can also provide clues to the diagnosis of CVT. A D-dimer level $<500 \mathrm{ng} / \mathrm{ml}$ had a negative predictive value of $99.6 \%$, but the positive predictive value was only $55.7 \%$. Therefore, a negative test makes CVT very unlikely, but the assay cannot be used to confirm 

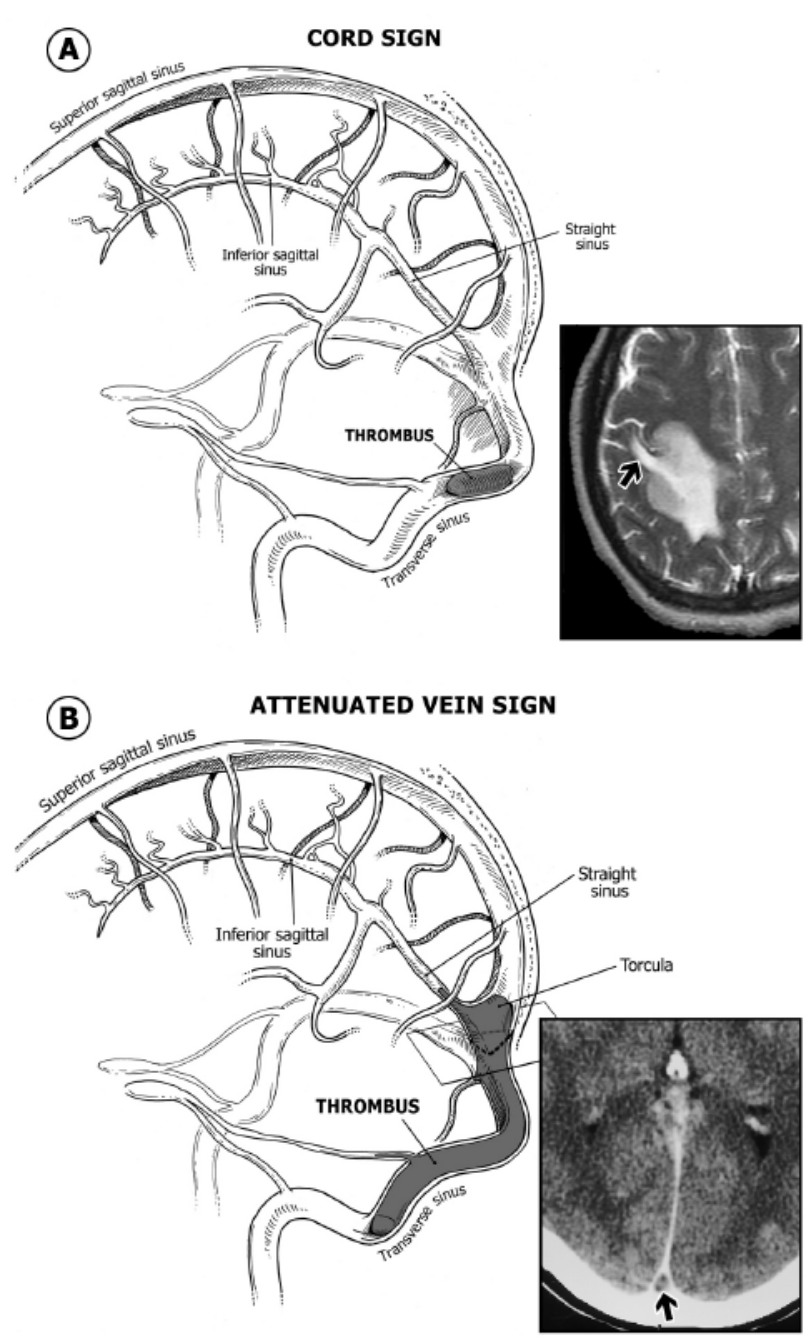

FIG. 3. Drawings and images. A: The cord sign as it appears in situ. Thrombus localized to an area within the sinus will correlate to homogeneous hyperattenuation on noncontrasted CT or hyperintensity on MR imaging (inset), as indicated (arrow). B: The attenuated vein sign in situ, demonstrating propagation of the thrombus into the deeper venous system. The empty delta sign is also shown as it appears on CT scanning (inset) with the site of thrombus indicated (arrow). (C2009 Medical College of Georgia

CVT due to other primary or coexisting causes for an elevated level..$^{31,52}$ The laboratory diagnosis of CVT remains a challenge, and physicians should move quickly to imaging techniques.

In a patient presenting to the emergency room with neurological symptoms, the most likely first test will be CT scanning. A thrombus in the superior sagittal sinus classically forms a "dense triangle" or rounded shape on noncontrast CT scans, and an "empty delta" after contrast injection, with enhancement around the clot likely due to inflammation..$^{8,44}$ This direct sign is seen in about onethird of patients. ${ }^{5}$ On MR imaging, the occluded sinus appears as a homogeneous hyperintense signal on T1- and T2-weighted images in a normal or bulging superior sagittal sinus. The CT scan may show gyral enhancement, pos- sibly due to blood-brain barrier breakdown, and regional hypoperfusion, edema, and hemorrhage in a nonarterial distribution are all clues to the diagnosis. ${ }^{43}$ Unfortunately, these are nonspecific, and the scans can reveal entirely normal findings in up to $30 \%$ of patients. Other, much rarer, signs that may reveal clues to the diagnosis are the cord sign and attenuated vein sign. A homogeneous area of hyperattenuation at sites of thrombosis on noncontrast CT scans is the cord sign, which indicates a thrombus within 7 days of formation. After 7-14 days, the thrombus becomes isodense and then hypodense on CT scans. Occasionally, the CT scan may not reveal findings and the cord sign may be seen on MR imaging as an area of hyperintensity representing a thrombosed vein. ${ }^{48}$ However, the sensitivity of the cord sign has been shown to be too low in excluding sinus thrombosis, and therefore, if CVT is suspected clinically, other methods should be undertaken to diagnose the condition, including CT angiography, MR imaging, or MR venography..$^{4,5,8,36,44,53,54}$ Deep veins, including the internal cerebral veins, great cerebral vein of Galen, straight sinus, or thalamostriate veins should be carefully analyzed, and the presence of hyperattenuation in the deep veins, known as the "attenuated vein sign," on noncontrast CT scanning is much more sensitive for CVT. The absence of it makes deep vein thrombosis highly unlikely. If hyperattenuation is present, an MR venography study should be performed immediately to confirm the diagnosis. Computed tomography venography has also been studied and is recommended for cases in which MR imaging is inconclusive, contraindicated, or unavailable. ${ }^{35,54}$ Figure 3 shows a schematic for what these signs would look like in situ. Figure 3A also shows an MR image of the cord sign as described by Sharma and Teoh, ${ }^{48}$ and Fig. 3B shows a depiction of the empty delta sign as seen on CT scanning, with a central area of hypoattenuation and surrounding enhancement.

The gold standard of CVT diagnosis is digital subtraction angiography. However, T1- and T2-weighted spin echo images showing thrombosis, together with MR venography showing a filling defect, represent valuable diagnostic tools and largely obviate the absolute need for angiography. The T2* echoplanar susceptibility-weighted images may be more helpful in the acute phase when the T1 signal is hypointense. This study is the most sensitive modality available to detect cortical vein thrombosis. Fluid attenuated inversion recovery is useful, especially in isolated venous occlusion where sensitivity of T1 and T2 signals is very low. Diffusion weighted imaging shows inconsistent patterns of edema, hemorrhage, and infarction within brain parenchyma. ${ }^{25}$ Most commonly, the diffusion coefficient is suggestive of vasogenic edema. Taken together, T1-, T2-, and T2*-weighted and FLAIR and DW imaging will detect most cases of CVT.7,36 One study found that MR imaging and MR angiography had a sensitivity of $100 \% .^{32}$

It is important to acknowledge that the same causes of thrombosis in the cerebral veins can cause clots in systemic veins as well. Investigation for deep vein thrombosis and pulmonary emboli is warranted, and these must also be watched for as complications following treatment for CVT. ${ }^{29}$ 


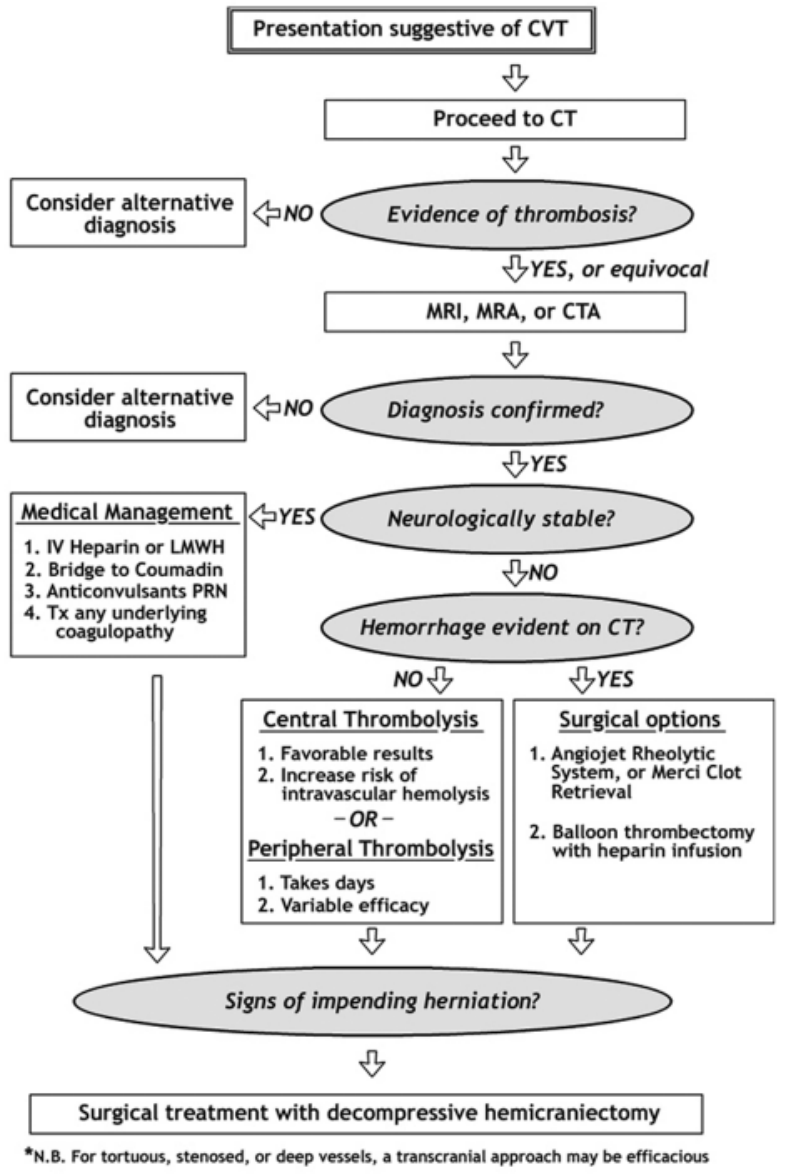

FIG. 4. Treatment algorithm for CVT. CTA = CT angiography; IV = intravenous; MRA = MR angiography; LMWH = low molecular-weight heparin; PRN = as needed. C2009 Medical College of Georgia

\section{Treatment}

\section{Medical Therapy}

Determining the best treatment plan for CVT can be difficult. Figure 4 depicts a suggested algorithm for selecting different treatments, driven by disease course and severity. Appropriate critical care measures should be undertaken in all patients, including intubation and intravenous fluids when needed, and seizure prophylaxis in those with risk factors, such as focal areas of edema or infarct. ${ }^{20}$ Although once an accepted treatment option, medical observation with fluids and prophylaxis alone has now been largely abandoned, as patients in this group uniformly did worse than those with greater measures. ${ }^{49}$

A few decades ago, only one therapy was widely available for CVT. Anticoagulation, typically with intravenous heparin, has been the mainstay of treatment since it was first used to treat CVT in 1941, and it remains the first-line therapy. Since clots themselves are thrombogenic, use of anticoagulation attenuates propagation, significantly reducing the burden that must be overcome by the venous system. ${ }^{41}$ Even in cases in which hemorrhage was detected, anticoagulation has repeatedly been shown to be safe and effective. A commonly used regimen is a bolus dose of up to $1000 \mathrm{U}$, followed by low-dose mainte-
J. N. Bentley, R. E. Figueroa, and J. R. Vender

nance therapy at 400-600 U/hour to an activated partial thromboplastin time of 60-70 seconds. ${ }^{49}$ Low-molecularweight heparin has also been studied, but controversy exists as to whether this is more efficacious than intravenous heparin. One study failed to show any improvement over intravenous heparin, ${ }^{17}$ while a meta-analysis reported an increased benefit of low-molecular-weight heparin and fewer bleeding complications. ${ }^{51}$ Low-molecular-weight heparin is generally administered at a dose of 180 antifactor $\mathrm{Xa} \mathrm{U} / \mathrm{kg} / 24$ hours in 2 subcutaneous injections per day. ${ }^{49}$

While heparin is effective in reducing clot formation, it is unable to lyse existing clots. Several options exist for more invasive treatments, and the choice to progress to these depends on failure of one treatment, as evidenced by clinical deterioration, lack of reasonably expected improvement, or signs of extensive venous compromise. ${ }^{49}$

Other methods of controlling elevated ICP are available and are used, such as steroids, acetazolamide, mannitol, or hyperventilation, but extreme caution must be maintained when administering these. ${ }^{5}$ In a prospective study comparing death or dependence at 6 months of follow-up, patients treated with steroids had the same outcomes as those not treated with steroids. In patients presenting without an intraparenchymal lesion on CT or MR imaging, treatment with steroids was associated with a worse prognosis. This outcome may be due to the prothrombotic properties of steroids or associated complications of treatment (for example, bleeding, infection, and hyperglycemia). Therefore, Level III evidence suggests that steroids are not beneficial unless they are being used to treat the underlying etiology (vasculitis, inflammation, infection, or malignancy), and are harmful in patients without preexisting parenchymal lesions. ${ }^{9}$

\section{Interventional Therapy}

Chemical endovascular thrombolysis with urokinase was first used in 1988, but this drug has since been largely replaced by $\mathrm{rtPA} .{ }^{33}$ Direct thrombolysis requires endovascular access, commonly with a Fogarty catheter inserted through the femoral or jugular vein. The thrombolytic agent is directly infused into the clot itself. The advantage to endovascular thrombolysis is its timely nature in reducing the clot. ${ }^{49}$ Some disagreement exists regarding durations of infusions at proper dosages. Interventionalists have reported using rtPA anywhere from 8 to 43 hours at total doses of 50-300 mg. ${ }^{47}$ The efficacy of rtPA has been studied in conjunction with heparin and has shown favorable results. Frey et al. ${ }^{23}$ observed complete flow restoration in $50 \%$ with $41.7 \%$ of patients having a complete recovery. Intracranial hemorrhage is one of the most feared complications of direct thrombolysis, and patients must be carefully selected for this technique, as it is believed to be associated with an increased risk of hemorrhage. ${ }^{33}$

Balloon thrombectomy has also been used, but this method is of limited value due to significant incidence of reocclusion. One method to reduce this risk is the use of an indwelling sinus catheter for maintenance of a continuous heparin infusion. Balloon thrombectomy has been associated with better clinical outcomes than endovascular thrombolysis or heparin alone and is especially 


\section{Diagnosis and treatment of cerebral vein thrombosis}

useful in preexisting hemorrhage. In a series analyzing 8 patients treated with balloon angioplasty, $88 \%$ showed signs of recovery. ${ }^{49}$

In patients in whom anticoagulation alone fails but in whom hemorrhage is a significant risk, mechanical thrombectomy offers a promising alternative treatment. The AngioJet Xpeedior (Possis Medical) is a rheolytic coaxial lumen guidewire-directed catheter that uses 6 high-velocity saline jets to create a pressure gradient. Pressurized saline is forced out of the catheter tip, causing a low-pressure region behind, to draw fragments of clot from the dural wall into the exhaust lumen, which can then be suctioned out of the body. Unlike traditional direct thrombolytic infusion, the process takes just minutes to recanalize the sinus and restore native flow, further promoting physiological fibrinolysis. ${ }^{15}$

Since the catheter initially must be advanced through the clot and then pulled back as the vacuum effect pulls the clot in, sinus stenosis could be a limiting factor. Additionally, the AngioJet catheter is relatively stiff, precluding navigation of tortuous veins or crossing into the deep venous system. To circumvent the limitation posed by difficult entry into deep veins, a transcranial approach has been successful. A small $(2-\mathrm{cm})$ craniotomy is formed at the inion, and the underlying confluence of sinuses is pierced using a micropuncture kit. The AngioJet catheter can then be inserted over a guidewire, and the deeper sinuses, such as the straight sinus, can be accessed directly. Gelfoam is used to seal the sinus, and the bone flap is replaced..$^{10,11,18,29,41,42,46}$ Mechanical thrombectomy with a rheolytic catheter resulted in clinical improvement in $75 \%$ of patients. One major advantage of this therapy is the immediate angiographic restoration of flow. ${ }^{28}$ The AngioJet system is associated with some complications, including intravascular hemolysis, ${ }^{19}$ dilutional anemia ${ }^{24}$ resulting from excess infusion of saline, and aspiration anemia due to suctioning of blood into the system. Clinicians must watch postoperatively for a decrease in the hematocrit level and clinical signs of hypovolemia, and they must transfuse as needed. ${ }^{24}$ Additionally, Curtin, et al. ${ }^{15} \mathrm{dem}-$ onstrated the usefulness of a combination of approaches in treating CVT. Balloon angioplasty, direct rtPA, and Angiojet thrombectomy were used due to lack of clinical improvement after 1 modality. The patient recovered, and the preexisting hemorrhage did not worsen.

Another attractive device that has been used is the Merci endovascular thrombus retrieval system (Concentric Medical, Inc.). This consists of a flexible nickel-titanium wire that assumes a helical conformation once out of the catheter tip. The tip is passed distal to the thrombus and is rotated while being retracted. The helix traps the clot, which can then be withdrawn. Suction can also be used to facilitate removal, and concomitant direct infusion of a thrombolytic can also be used. An advantage of the Merci device over the AngioJet is its maneuverability, but it does carry an increased risk of vessel injury. However, most studies evaluating this risk were performed in arterial thrombectomy, and risk of injury to the tougher dural wall is believed to be lower. These new devices were themselves preceded by various clot-removal systems, and research continues to make improvements on currently available ones. Physicians must maintain an awareness of continued advances in the field..$^{12,41}$

\section{Surgical Therapy}

At the extreme end of the clinical continuum are patients who present with signs of impending herniation. Here, venous occlusion results in significantly increased ICP that cannot be compensated for by collateral flow or $\mathrm{CSF}$ reduction. In these cases, any delay in therapy leads to imminent death, and the mortality rate is, as expected, considerably higher. Endovascular approaches might not alleviate enough of the burden as quickly as needed, and a useful validated approach is decompressive hemicraniectomy. Most of what is known about the efficacy of hemicraniectomy is based on various case reports done over the last decade. Although the reports are relatively few, this option resulted in favorable outcome in $11(84.6 \%)$ of 13 cases. Several patients in these reports had clinical signs of herniation, with fixed pupils and/or coma, and had reversal of these and complete functional recovery. Suggested indications include a unilateral dilated and fixed pupil; rapid deterioration in the Glasgow Coma Scale score; and significant midline shift or elimination of basal cisterns on CT or MR imaging. The procedure creates a generous hemicraniectomy with the dura incised widely. However, bias may exist toward reporting of successful cases, and the need for a prospective analysis of this intervention cannot be overlooked. Preoperative and postoperative anticoagulation should also be used here as well. ${ }^{2,14,50,55}$ Keller et al. ${ }^{27}$ reported 4 cases of decompressive hemicraniectomy for malignant CVT for which preoperative anticoagulation was discontinued. The anticoagulation was restarted at a half dose 12 hours after surgery and increased to the full dose at 24 hours postoperatively. No patient experienced exacerbation of preexisting hemorrhage or other bleeding. A larger study should be done, however, to understand the optimal perioperative management and clinical need for anticoagulation.

\section{Follow-Up}

Appropriate follow-up consists of ensuring that underlying causes for cerebral venous thrombosis are investigated, and any reversible cause of coagulopathy is treated. Additionally, patients should be assessed for continued recanalization with imaging. About $15 \%$ of patients will have no identifiable cause, but some causes can be easily ruled out. Females are affected almost 3 times as often as males, and pregnancy, oral contraceptive use, or puerperium can be easily evaluated. The myriad causes of hypercoagulable states should be worked up with appropriate laboratory tests to ensure success and prevent recurrence. The cause is also important for determining the duration of oral anticoagulation therapy following bridging from intravenous therapy. For reversible states the usual range is 3-6 months, while in inherited disorders, the treatment is lifelong. ${ }^{7,15,49}$ Appropriate follow-up includes venous imaging after $\sim 2$ weeks with MR imaging and MR angiography unless MR imaging alone shows sustained thrombosis. Images suggest recanalization by decreased 
or absent signal on T1- and T2-weighted MR images, and normal sinus anatomy on MR angiography. ${ }^{32}$

Most patients $(80 \%)$ who were independent before CVT return to independence following the event. However, many will report long-term sequelae. The most common complaint, reported in $75 \%$ of patients, was decreased inability to concentrate that affected psychosocial and work-related activities. About half of the patients can be expected to report headache, while approximately one-third will have increased fatigue or depression. It is important for clinicians to realize that CVT patients may follow-up with long-term complaints, and these should be appropriately addressed.,30

\section{Conclusions}

Due to the rarity of the condition, with only $1-5$ people per million being diagnosed with CVT, it is often overlooked in the differential of causes for neurological deficits. ${ }^{7}$ However, without treatment, mortality rates are high, and in an age of sophisticated imaging modalities, the consequence of not considering CVT is of great detriment to the patient. ${ }^{10}$ Neurological manifestations are extremely varied in course and severity, and even cases of isolated headache can be CVT. Therefore, physicians must maintain a high degree of suspicion when dealing with nonspecific neurological complaints. ${ }^{5-8,10,13,16,20,21,36}$ Confirmatory imaging can be obtained with MR imaging and MR venography; CT scanning may provide some clues, but is not as useful in making the definitive diagnosis. . $^{3,736}$

Treatment of CVT is an additional source of confusion. Most agree that systemic intravenous anticoagulation is an appropriate first-line therapy, even in the presence of intracranial hemorrhage, and this will be sufficient for the majority of cases. ${ }^{41,49}$ The decision on how to best to proceed in treating patients stems from clinical or radiological severity. The presence of poor prognostic risk factors, rapid deterioration, failure of a treatment modality, significant clot burden, or signs of impending herniation warrant more aggressive and invasive treatments. ${ }^{49}$ The presence or absence of preexisting intracranial hemorrhage determines the risk of direct endovascular thrombolysis. Mechanical thrombectomy, with or without thrombolysis, presents an attractive option in cases of hemorrhage that has shown promising results. Borrowing from devices already in use for other sites of vessel occlusion, such as coronary and cerebral arteries, new techniques for mechanical venous thrombectomy are quickly emerging. ${ }^{12,15,17,41}$ These systems can be applied transfemorally, transjugularly, or, if tortuous or deep veins are involved, transcranially via the confluence of sinuses. ${ }^{10}$ Hemicraniectomy is an option for those patients facing herniation. ${ }^{2,14,50,55}$ Adjuncts to these should also be included when appropriate, such as use of balloons, mannitol, or hyperventilation. ${ }^{49}$ Diagnosis and treatment of CVT may present challenges, but rapid and aggressive management when needed will result in low mortality and patient recovery without lifestyle-limiting sequelae.

\section{Disclaimer}

The authors report no conflict of interest concerning the materials or methods used in this study or the findings specified in this paper.

\section{References}

1. Aaron S, Alexander M, Thomas M, George B, Mammen J: Cerebral venous thrombosis due to homozygous factor V Leiden mutation. Neurol India 57:91, 2009

2. Becker KJ, Brott TG: Approval of the MERCI clot retriever: a critical view. Stroke 36:400-403, 2005

3. Bender A, Schulte-Altedorneburg G, Mayer TE, Pfefferkorn T, Birnbaum T, Feddersen B, et al: Functional outcome after severe cerebral venous thrombosis. J Neurol 254:465-470, 2007

4. Boukobza M, Crassard I, Bousser MG: When the "dense triangle" in dural sinus thrombosis is round. Neurology 69:808, 2007

5. Bousser MG: Cerebral venous thrombosis: diagnosis and management. J Neurol 247:252-258, 2000

6. Bousser MG, Chiras J, Bories J, Castaigne P: Cerebral venous thrombosis-a review of 38 cases. Stroke 16:199-213, 1985

7. Bousser MG, Ferro JM: Cerebral venous thrombosis: an update. Lancet Neurol 6:162-170, 2007

8. Buonanno FS, Moody DM, Ball MR, Laster DW: Computed cranial tomographic findings in cerebral sinovenous occlusion. J Comput Assist Tomogr 2:281-290, 1978

9. Canhão P, Cortesão A, Cabral M, Ferro JM, Stam J, Bousser MG: Are steroids useful to treat cerebral venous thrombosis? Stroke 39:105-110, 2008

10. Chahlavi A, Steinmetz MP, Masaryk TJ, Rasmussen PA: A transcranial approach for direct mechanical thrombectomy of dural sinus thrombosis. Report of two cases. J Neurosurg 101:347-351, 2004

11. Chaloupka JC, Mangla S, Huddle DC: Use of mechanical thrombolysis via microballoon percutaneous transluminal angioplasty for the treatment of acute dural sinus thrombosis: case presentation and technical report. Neurosurgery 45:650-657, 1999

12. Chow K, Gobin YP, Saver J, Kidwell C, Dong P, Viñuela F: Endovascular treatment of dural sinus thrombosis with rheolytic thrombectomy and intra-arterial thrombolysis. Stroke 31:1420-1425, 2000

13. Cortez O, Schaeffer CJ, Hatem SF, Glauser J, Ahmed M: Cases from the Cleveland Clinic: cerebral venous sinus thrombosis presenting to the emergency department with worst headache of life. Emerg Radiol 16:79-82, 2009

14. Coutinho JM, Majoie CB, Coert BA, Stam J: Decompressive hemicraniectomy in cerebral sinus thrombosis: consecutive case series and review of the literature. Stroke 40:2233-2235, 2009

15. Curtin KR, Shaibani A, Resnick SA, Russell EJ, Simuni T: Rheolytic catheter thrombectomy, balloon angioplasty, and direct recombinant tissue plasminogen activator thrombolysis of dural sinus thrombosis with preexisting hemorrhagic infarctions. AJNR Am J Neuroradiol 25:1807-1811, 2004

16. de Bruijn SF, de Haan RJ, Stam J: Clinical features and prognostic factors of cerebral venous sinus thrombosis in a prospective series of 59 patients. For The Cerebral Venous Sinus Thrombosis Study Group. J Neurol Neurosurg Psychiatry 70:105-108, 2001

17. de Bruijn SF, Stam J: Randomized, placebo-controlled trial of anticoagulant treatment with low-molecular-weight heparin for cerebral sinus thrombosis. Stroke 30:484-488, 1999

18. Dowd CF, Malek AM, Phatouros CC, Hemphill JC III: Application of a rheolytic thrombectomy device in the treatment of dural sinus thrombosis: a new technique. AJNR Am J Neuroradiol 20:568-570, 1999 
19. Dukkipati R, Yang EH, Adler S, Vintch J: Acute kidney injury caused by intravascular hemolysis after mechanical thrombectomy. Nat Clin Pract Nephrol 5:112-116, 2009

20. Einhäupl K, Bousser MG, de Bruijn SF, Ferro JM, Martinelli I, Mashur F, et al: EFNS guideline on the treatment of cerebral venous and sinus thrombosis. Eur J Neurol 13:553-559, 2006

21. Ferro JM, Canhão P, Stam J, Bousser MG, Barinagarrementeria F: Prognosis of cerebral vein and dural sinus thrombosis: results of the International Study on Cerebral Vein and Dural Sinus Thrombosis (ISCVT). Stroke 35:664-670, 2004

22. Ferro JM, Lopes MG, Rosas MJ, Fontes J: Delay in hospital admission of patients with cerebral vein and dural sinus thrombosis. Cerebrovasc Dis 19:152-156, 2005

23. Frey JL, Muro GJ, McDougall CG, Dean BL, Jahnke HK: Cerebral venous thrombosis: combined intrathrombus rtPA and intravenous heparin. Stroke 30:489-494, 1999

24. Fries G, Wallenfang T, Hennen J, Velthaus M, Heimann A, Schild $\mathrm{H}$, et al: Occlusion of the pig superior sagittal sinus, bridging and cortical veins: multistep evolution of sinus-vein thrombosis. J Neurosurg 77:127-133, 1992

25. Idbaih A, Boukobza M, Crassard I, Porcher R, Bousser MG, Chabriat H: MRI of clot in cerebral venous thrombosis: high diagnostic value of susceptibility-weighted images. Stroke 37:991-995, 2006

26. Ilhan D, Gulcan E, Uzuner N, Celikkas E: Cerebrovascular manifestations of Behçet's disease. J Clin Neurosci 16:576578, 2009

27. Keller E, Pangalu A, Fandino J, Könü D, Yonekawa Y: Decompressive hemicraniectomy in severe cerebral venous and dural sinus thrombosis. Acta Neurochir Suppl 94:177-183, 2005

28. Kikuta K, Miyamoto S, Kataoka H, Yamada K, Takagi Y, Nozaki K, et al: An adult case of moyamoya syndrome that developed dural sinus thrombosis associated with protein $\mathrm{C}$ deficiency: case report and literature review. Surg Neurol 63:480-484, 2005

29. Kirsch J, Rasmussen PA, Masaryk TJ, Perl J II, Fiorella D: Adjunctive rheolytic thrombectomy for central venous sinus thrombosis: technical case report. Neurosurgery 60:E577E578, 2007

30. Koopman K, Uyttenboogaart M, Vroomen PC, van der Meer J, De Keyser J, Luijckx GJ: Long-term sequelae after cerebral venous thrombosis in functionally independent patients. J Stroke Cerebrovasc Dis 18:198-202, 2009

31. Kosinski CM, Mull M, Schwarz M, Koch B, Biniek R, Schläfer $\mathrm{J}$, et al: Do normal D-dimer levels reliably exclude cerebral sinus thrombosis? Stroke 35:2820-2825, 2004

32. Lafitte F, Boukobza M, Guichard JP, Hoeffel C, Reizine D, Ille O, et al: MRI and MRA for diagnosis and follow-up of cerebral venous thrombosis (CVT). Clin Radiol 52:672-679, 1997

33. Lewis MB, Bousser MG: Cerebral venous thrombosis: nothing, heparin, or local thrombolysis? Stroke 30:481-483, 1999

34. Lin A, Foroozan R, Danesh-Meyer HV, De Salvo G, Savino PJ, Sergott RC: Occurrence of cerebral venous sinus thrombosis in patients with presumed idiopathic intracranial hypertension. Ophthalmology 113:2281-2284, 2006

35. Linn J, Pfefferkorn T, Ivanicova K, Müller-Schunk S, Hartz $\mathrm{S}$, Wiesmann M, et al: Noncontrast CT in deep cerebral venous thrombosis and sinus thrombosis: comparison of its diagnostic value for both entities. AJNR Am J Neuroradiol 30:728-735, 2009

36. Masuhr F, Mehraein S, Einhäupl KJ: Cerebral venous and sinus thrombosis. J Neurol 251:11-23, 2004

37. Mert E, Ozge A, Taşdelen B, Yilmaz A, Bilgin NG: What clues are available for differential diagnosis of headaches in emergency settings? J Headache Pain 9:89-97, 2008

38. Meyohas MC, Roullet E, Rouzioux C, Aymard A, Pelosse B, Eliasceiwicz M, et al: Cerebral venous thrombosis and dual primary infection with human immunodeficiency virus and cytomegalovirus. J Neurol Neurosurg Psychiatry 52:10101011, 1989

39. Nair V, Mohapatro AK, Sreedhar M, Indrajeet IK, Tewari AK, Anand AC, et al: A case of hereditary protein S deficiency presenting with cerebral sinus venous thrombosis and deep vein thrombosis at high altitude. Acta Haematol 119:158161,2008

40. Nakase H, Heimann A, Kempski O: Local cerebral blood flow in a rat cortical vein occlusion model. J Cereb Blood Flow Metab 16:720-728, 1996

41. Newman CB, Pakbaz RS, Nguyen AD, Kerber CW: Endovascular treatment of extensive cerebral sinus thrombosis. Case report. J Neurosurg 110:442-445, 2009

42. Opatowsky MJ, Morris PP, Regan JD, Mewborne JD, Wilson JA: Rapid thrombectomy of superior sagittal sinus and transverse sinus thrombosis with a rheolytic catheter device. AJNR Am J Neuroradiol 20:414-417, 1999

43. Rao KC, Knipp HC, Wagner EJ: Computed tomographic findings in cerebral sinus and venous thrombosis. Radiology 140:391-398, 1981

44. Rodallec MH, Krainik A, Feydy A, Hélias A, Colombani JM, Jullès MC, et al: Cerebral venous thrombosis and multidetector CT angiography: tips and tricks. Radiographics 26:S5S18, 2006

45. Sakamoto S, Akutsu K, Kawase K, Takada T, Seyama H, Takahashi J, et al: Simultaneous presentations of deep vein thrombosis and cerebral sinus thrombosis in a case of primary antiphospholipid syndrome. Angiology 59:765-768, 2008

46. Scarrow AM, Williams RL, Jungreis CA, Yonas H, Scarrow MR: Removal of a thrombus from the sigmoid and transverse sinuses with a rheolytic thrombectomy catheter. AJNR Am J Neuroradiol 20:1467-1469, 1999

47. Scott JA, Pascuzzi R, Hall P, Becker G: Treatment of dural sinus thrombosis with local urokinase infusion. J Neurosurg 68:284-287, 1988

48. Sharma VK, Teoh HL: Isolated cortical vein thrombosis-the cord sign. Neuroradiology 3:21-24, 2009

49. Soleau SW, Schmidt R, Stevens S, Osborn A, MacDonald JD: Extensive experience with dural sinus thrombosis. Neurosurgery 52:534-544, 2003

50. Stefini R, Latronico N, Cornali C, Rasulo F, Bollati A: Emergent decompressive craniectomy in patients with fixed dilated pupils due to cerebral venous and dural sinus thrombosis: report of three cases. Neurosurgery 45:626-629, 1999

51. Van Dongen CJ, van den Belt AGM, Prins MH, Lensing AWA: Fixed dose subcutaneous low molecular weight heparins versus adjusted dose unfractionated heparin for venous thromboembolism. Cochrane Database Syst Rev 18:CD001100, 2004

52. Vatankhah B, Fürst A, Schlachetzki F: Do normal D-dimer levels reliably exclude cerebral sinus thrombosis? A solution of problems? Stroke 36:2528-2529, 2005

53. Vijay RK: The cord sign. Radiology 240:299-300, 2006

54. Virapongse C, Cazenave C, Quisling R, Sarwar M, Hunter S: The empty delta sign: frequency and significance in 76 cases of dural sinus thrombosis. Radiology 162:779-785, 1987

55. Zeng L, Derex L, Maarrawi J, Dailler F, Cakmak S, Nighoghossian $\mathrm{N}$, et al: Lifesaving decompressive craniectomy in 'malignant' cerebral venous infarction. Eur J Neurol 14:e27e28, 2007

Manuscript submitted July 15, 2009.

Accepted August 26, 2009.

Address correspondence to: John R. Vender, M.D., Department of Neurosurgery, BI 3088, 1120 15th Street, Augusta, Georgia 30912. email: jvender@mail.mcg.edu. 